\title{
Noninvasive ventilation in stable chronic obstructive pulmonary disease
}

\section{To the Editor:}

Chronic obstructive pulmonary disease (COPD) with severe emphysema is a continuously progressing disease, resulting in respiratory failure. Pharmacological treatment may be optimised in the last years, but only the introduction of long-term oxygen treatment has revealed an improvement in survival for patients with advanced COPD [1].

Noninvasive positive pressure ventilation (NPPV) is a new approach to directly correct ventilatory insufficiency. The beneficial effects of NPPV during acute exacerbation of COPD are well documented [2], and NPPV is frequently applied in these patients today [3]. However, domiciliary longterm use of NPPV by patients with severe COPD remains controversial.

In 1996, the Italian Rehabilitation and Chronic Care Study Group started a prospective, randomised, controlled, multicentre trial to evaluate NPPV in severe chronic stable COPD. The main outcome parameters were arterial blood gases, hospital and intensive care unit admissions and length of stay, health related quality of life, and exercise tolerance. The results of this study had been recently presented in the European Respiratory Journal [4]. The conclusions drawn are not in favour of long-term use of NPPV in severe COPD patients. Apart from a quality of life assessment (Maugeri Foundation Respiratory Questionnaire (MRF-28)), none of the other outcome parameters could be significantly improved. The reason for this should be sought in the "ventilator setting" instructions of the protocol: ventilator settings were titrated individually for each patient with the target of maximally tolerated inspiratory pressures and expiratory pressures in the range of $2-5 \mathrm{~cm} \cdot \mathrm{H}_{2} \mathrm{O}$. However, a reduction of baseline carbon dioxide arterial tension by $\geqslant 5 \%$ during $\geqslant 1 \mathrm{~h}$ of daytime NPPV had been considered an effective treatment (E. Clini, Fondazione Villa Pineata ONLUS, Italy, personal communication). This resulted in a mean inspiratory pressure of $14 \pm 3 \mathrm{~cm} \cdot \mathrm{H}_{2} \mathrm{O}$ and a mean expiratory pressure of $2 \pm 1 \mathrm{~cm} \cdot \mathrm{H}_{2} \mathrm{O}$.

The findings of the study by CLINI et al. [4] are in accordance with the survival and hospital admission rates reported by CASANOVA et al. [5], who applied NPPV in a similar cohort of COPD patients with a mean inspiratory pressure of $12 \mathrm{~cm} \cdot \mathrm{H}_{2} \mathrm{O}$ and a mean expiratory pressure of $4 \mathrm{~cm} \cdot \mathrm{H}_{2} \mathrm{O}$. However, in $1995 \mathrm{MeECHAM-JONES} \mathrm{et} \mathrm{al.} \mathrm{[6]} \mathrm{demon-}$ strated significant improvements of blood gases, sleep quality and quality of life in hypercapnic COPD patients after 3 months of NPPV with a mean inspiratory pressure of $20 \mathrm{~cm} \mathrm{H} \mathrm{H}_{2} \mathrm{O}$.

It is reasonable to speculate that the applied pressures in the study by MEECHAM-JONES et al. [6] resulted in better gas exchange and unloading of the ventilatory muscles. NPPV might otherwise be "underdosed" in patients with severe COPD and the therapeutic goal of correcting the underlying pathophysiology is missed. It can be troublesome to establish NPPV in patients with severe COPD and with growing ventilatory pressures it becomes more and more difficult to keep the patient compliant. The balance between therapeutic requirements and patients' comfort is a challenge for both patient and staff.

Further studies confirming and extending the findings of MeEcham-Jones et al. [6] findings to define the role of noninvasive positive pressure ventilation as a treatment option for patients with severe chronic obstructive pulmonary disease are needed.

\author{
T. Köhnlein, T. Welte \\ Otto-von-Guericke-Universität Magdeburg, Pulmonary and \\ Intensive Care Medicine, Leipziger Strasse 44, D-39120 Mage- \\ burg, Germany.
}

\section{References}

1. Report of the Medical Research Council Working Party. Long-term domiciliary oxygen in chronic corpulmonale complicating chronic bronchitis and emphysema. Lancet 1981; 1: 681-685.

2. Brochard L, Mancebo J, Wysocki M, et al. Noninvasive ventilation for acute exacerbations of chronic obstructive pulmonary disease. $N$ Engl J Med 1995; 333: 817-822.

3. Keenan SP, Kernerman PD, Cook DJ, Martin CM, McCornmack D, Sibbald WJ. Effect of noninvasive positive pressure ventilation on mortality in patients admitted with acute respiratory failure: A meta-analysis. Crit Care Med 1997; 25: 1685-1692.

4. Clini E, Sturani C, Rossi A, et al. The Italian mulitcentre study on noninvasive ventilation in chronic obstructive pulmonary disease patients. Eur Respir J 2002; 20: 529-538.

5. Casanova C, Celli BR, Tost L, et al. Long-term controlled trial of nocturnal nasal positive pressure ventilation in patientes with severe COPD. Chest 2000; 118: 1582-1590.

6. Meecham Jones DJ, Paul EA, Jones PW, Wedzicha JA. Nasal pressure support ventilation plus oxygen compared with oxygen therapy alone in hypercapnic COPD. Am J Respir Crit Care Med 1995; 152: 538-544.

\section{From the authors:}

We would like to thank T. Koehnlein and T. Welte for their comments on our paper dealing with the use of noninvasive positive pressure ventilation (NPPV) in chronic obstructive pulmonary disease (COPD) patients as recently published in the European Respiratory Journal [1].

They essentially stated that the results of that multicentre study were negative, by observing that the lack of response in most of the physiological and clinical variables could be ascribed to the "relatively" low level of assistance delivered, as a mean, by the ventilator.

However, we keep on thinking about a possible positive role for NPPV in a clearly selected sample of severe COPD patients. Indeed, the long-term protective effect on blood gases (namely, arterial carbon dioxide tension $\left(\mathrm{Pa}, \mathrm{CO}_{2}\right)$ ) while breathing oxygen (which is the most common daily condition for those patients) observed in the NPPV group has indirectly shown the actual physiological value of this study [1]. Although the pathophysiological reason for this behaviour (different from that observed in the control patients) cannot be drawn from this clinical study, it is reasonable to hypothesise that a probable long-term protection against hypoventilation, as demonstrated previously [2], also still occurred at a lower level of assistance.

Nevertheless, we completely agree with T. Koehnlein and $\mathrm{T}$. Welte in that increasing pressure levels might decrease compliance and affect the physiological response (mainly related to the increasing leaks) of the ventilatory treatment. 
Finally, we feel confident that the positive result of the Maugeri Foundation Respiratory Failure item set (MRF-28) questionnaire [3] assessment in the treated subjects, also showed a positive impact of one of the most important endpoints of the long-term ventilatory strategy in these patients, which is the health-related quality of life (HRQoL). Although, it is still not clear whether survival can be positively influenced by NPPV in addition to standard long-term oxygen therapy (LTOT), the result obtained on specific HRQoL (together with the positive, although not significant, trend towards the reduction in the follow-up admissions in the critical care area) seems to justify the prescripton of NPPV, at least when a poor condition of the patient (i.e. severe disability, frequent exacerbations and hospitalisations, low effect and clinical control by LTOT) can be proven.

Obviously, any conclusion should be made with caution at present. However, although the prescription of noninvasive positive pressure ventilation cannot be recommended as a generalised option in the chronic obstructive pulmonary disease patients, an effort should be made to look for the best candidates on the basis of their history and the pathophysiological background available.
E. Clini*, N. Ambrosino

*Division of Pulmonary Rehabilitation, Fondazione Villa Pineta ONLUS, Pavullo and ${ }^{\#}$ Cardiosurgery Dept, Division of Pneumology, Azienda Ospedaliera Pisana, Pisa, Italy.

\section{References}

1. Clini E, Sturani C, Rossi A, et al. on behalf of the Rehabilitation and Chronic Care Study Group, Italian Association of Hospital Pulmonologisis (AIPO). The Italian mulitcentre study on noninvasive ventilation in chronic obstructive pulmonary disease patients. Eur Respir $J$ 2002; 20: $529-538$.

2. Meecham Jones DJ, Paul EA, Jones PW, Wedzicha JA. Nasal pressure support ventilation plus oxygen compared with oxygen therapy alone in hypercapnic COPD. $\mathrm{Am}$ J Respir Crit Care Med 1995; 152: 538-544, 1327.

3. Carone M, Bertolotti G, Anchisi F, et al. Analysis of factors that characterize health impairment in patients with chronic respiratory failure. Eur Respir J 1999; 13: 1293-1300.

\section{Survival in COPD patients after regular use of fluticasone propionate and salmeterol}

\section{To the Editor:}

I read with interest the paper by SORIANO et al. [1], in which the authors stated that fluticasone proprionate alone or in combination with salmeterol is associated with increased survival of chronic obstructive pulmonary disease (COPD) patients managed in primary care.

First, according to their data, the authors should have considered replacing fluticasone with inhaled steroids. More importantly, they did not discuss potential limitations that could have cast doubt on this statement.

Some of the data presented in table 1 of this paper pointed towards potential important differences between the three "active" groups and the reference group at baseline. Indeed, baseline treatment in the three groups receiving fluticasone and/or salmeterol could be described as fairly typical for moderate-to-severe COPD patients, with $>90 \%$ of the patients receiving inhaled bronchodilators. In contrast, less than onehalf of the reference group used inhaled bronchodilators at baseline, which is also clearly different from the reality of drug use in COPD in the UK [2]. This is peculiar for a group of COPD patients that show a high mortality. The $25 \%$ mortality at $2 \mathrm{yrs}$ in this group parallels the prognosis of COPD patients requiring long-term oxygen therapy [3, 4] or with a forced expiratory volume in one second (FEV1) of $<30 \%$ predicted in the intermittent positive pressure breathing trial [5].

Moreover, patients in the reference group were significantly older, had significantly more comorbidities (the combination of the two pointing towards potentially more severe comorbidities), and were less likely to have severe COPD as defined by the authors (use of oxygen or nebulised therapy).

Finally, the cause of death was not reported.

These points lead to the following questions being raised. 1) Could the method of inclusion in the UK General Practice Research Database (GPRD) explain these differences? Neither the description by the authors nor previous papers related to the GPRD [6-8] clarify whether inclusion in the database as a COPD patient required contact with a general practitioner (GP) for primary diagnosis of COPD or whether the mention of COPD at a contact for another purpose (and therefore as a secondary diagnosis) was sufficient. Since COPD is prevalent, particularly so in an old population such as the reference group of SORIANO et al. [1] (mean age \pm SD $72 \pm 10 \mathrm{yrs}$ ) [9], the detection of COPD as a secondary diagnosis when contact is made with the GP should not be a rare occurrence. Accordingly, the primary reason for the healthcare contact leading to the inclusion in the GPRD could have been a comorbid state with a worse prognosis. This could play a role in the higher all-cause mortality observed in the reference group. 2) The authors reported the sensitivity of a diagnosis of COPD in the GPRD but did not report the specificity, which is equally important in the interpretation of their data. As patients from the three active groups seem to be quite typical COPD populations, could patients misdiagnosed as COPD be overrepresented in the reference group? 3) Since other studies based on the GPRD were related to the cause of death recorded in the database [10,11], could the authors have clarified whether the cause of death was available in the different groups?

\section{E. Marchand}

Service de Pneumologie, Cliniques Universitaires UCL de Mont-Godinne, B-5530 Yvoir, Belgium.

\section{References}

1. Soriano JB, Vestbo J, Pride NB, Kiri V, Maden C, Maier WC. Survival in COPD patients after regular use of fluticasone propionate and salmeterol in general practice. Eur Respir J 2002; 20: 819-825. 